\title{
Fabry-Pérot cavities based on chemical etching for high temperature and strain sensing
}

\author{
Paula A. R. Tafulo, ${ }^{\mathrm{a}, \mathrm{b}}$, P. A. S. Jorge ${ }^{\mathrm{a}}$, J. L. Santos ${ }^{\mathrm{a}, \mathrm{b}}$, O. Frazão ${ }^{\mathrm{a}}$ \\ ${ }^{a}$ INESC-Porto, UOSE, Rua do Campo Alegre, 687, 4150-179 Porto, Portugal. \\ ${ }^{\mathrm{b}}$ University of porto, Department of Physics, Rua do Campo Alegre, 687, 4150-179 Porto, Portugal.
}

\begin{abstract}
In this paper, two novel hybrid multimode/single mode fiber Fabry-Pérot (FP) cavities were compared. The cavities fabricated by chemical etching are presented as high temperature and strain sensors. In order to produce this FP cavity a single mode fiber was spliced to a graded index multimode fiber with $62.5 \mu \mathrm{m}$ core diameter. The multimode fiber was cut approximately $150 \mu \mathrm{m}$ away from the splice. Then the tip of the fiber containing the multimode fiber segment was dipped into a solution of $48 \%$ of HF during 8 minutes, creating a concavity due to the fact that the reaction between HF and the germanium doped fiber core is much faster than the reaction between HF and the pure silica cladding. By this method a concavity of approximately $100 \mu \mathrm{m}$ deep was created at the fiber tip. Two different FP cavities can be fabricated. The first cavity is obtained when a spliced with an identical tip concavity fiber (Sensor A) and the second is created when a tip concavity is spliced to a single mode fiber (Sensor B). The Fabry-Pérot cavities were tested as a high temperature sensor in the range between room temperature and $800^{\circ} \mathrm{C}$ and as strain sensors. A reversible shift of the interferometric peaks with temperature allowed to estimate a sensitivity of $0.75 \pm 0.03 \mathrm{pm} /{ }^{\circ} \mathrm{C}$ and $0.98 \pm 0.04 \mathrm{pm} /{ }^{\circ} \mathrm{C}$ for the sensor A and B respectively. For strain measurement sensor A demonstrated a sensitivity of $1.85 \pm 0.07 \mathrm{pm} / \mu \varepsilon$ and sensor B showed a sensitivity of $3.14 \pm 0.05 \mathrm{pm} / \mu \varepsilon$. The sensors demonstrated the feasibility of low cost fiber optic sensors for high temperature and strain.
\end{abstract}

Keywords: Fiber optic sensors, strain, temperature, Fabry-Pérot interferometer, multimode optical fiber.

\section{INTRODUCTION}

Fiber optic sensors based in Fabry-Pérot (FP) cavities are attractive due to their high sensitivity to pressure, strain, temperature and acoustic waves. Fiber optic interferometric sensors have numerous advantages over conventional sensors such as immunity to electric interference, response to a wide variety of measurands, high resolution, high accuracy, small size, etc. Fiber optic interferometric sensors have been applied in health monitoring, airplanes, space aircrafts, civil engineering structures [1].

FP cavities can be extrinsic or intrinsic to the fiber. Intrinsic Fabry-Pérot interferometric (IFPI) sensors have a cavity within the fiber. Having the sensing element in the fiber overcomes some of the disadvantages of extrinsic sensors. Tipically, IFPI sensors where mirror are not used, have low reflectivity due to the small refractive index differences at the cavity interfaces [3]. There are many different techniques to create intrinsic fiber optic FP cavities, such as those based in a pair of splices [4], a pair of tapers [5], fiber Bragg gratings, either using two consecutive identical grating structures [6] or a single low reflectivity FBG and the fiber-end Fresnel reflection [7] .

High temperature strain gauges are one of the most important applications of FP sensors. Electrical strain gauges are a mature technology and are widely used. However, the major drawbacks of these sensors such as short lifetime under high temperature, nonlinear distortion and vulnerability to electromagnetic interference, can be overcome by fiber optic strain

International Conference on Applications of Optics and Photonics, edited by Manuel F. M. Costa,

Proc. of SPIE Vol. 8001, 800141 - @ 2011 SPIE - CCC code: 0277-786X/11/\$18 · doi: 10.1117/12.892090

Proc. of SPIE Vol. $8001800141-1$ 
sensors. The last are capable to operate in harsh environments, with high resolution and immunity to electromagnetic interference [8].

Low finesse fiber FP interferometers are commonly used as optical sensors for measurements of physical parameters as strain, temperature, refractive index, pressure, etc. Normally these sensors have sealed cavities [9-11]. Several authors have used the chemical etching technique to create sensors based in Fabry-Pérot cavities. Sensor geometries based in chemical etching technique to measure strain and temperature [11], refractive index [10] and pressure [14], were reported.

In this work two novel Fabry-Pérot interferometers based on chemical etching in multimode graded index fibers are fabricated and their response to temperature and strain are compared.

\section{FP-CAVITY FABRICATION}

\subsection{Fabrication of the FP cavities}

Chemical etching is a simple and inexpensive method to create concave or convex fiber tips, still allowing high optical transmission. Chemical etching of an optical fiber tip consists in dipping the tip of the fiber in hydrofluoric acid (HF). Controlling the time, temperature and agitation it is possible to create concave or sharpened fiber tips [12]. The overall chemical etching reaction, dissolution of silicon dioxide, also kwon as silica ( $\mathrm{SiO} 2)$, in aqueous HF solution, can be described as,

$$
\mathrm{SiO}_{2}+6 \mathrm{HF} \rightarrow \mathrm{H}_{2} \mathrm{SiF}_{6}+2 \mathrm{H}_{2} \mathrm{O}
$$

The fused silica dissolution in HF is influenced by the presence and composition of dopants. In case of an optical fiber with a germanium $(\mathrm{Ge})$ doped core and a silica cladding, the Ge core etches faster than the silica based cladding. Germanium oxide $(\mathrm{GeO} 2)$ has, as fused silica, a tetrahedral configuration. For $\mathrm{Ge}-\mathrm{O}$ the energy bond dissociation is lower than for Si-O, $662 \mathrm{~kJ} / \mathrm{mol}$ and $799 \mathrm{~kJ} / \mathrm{mol}$, respectively. Fused silica doped with germanium can be represented as ( $\mathrm{Si}-\mathrm{Ge})$ and $(\mathrm{Ge}-\mathrm{Ge})$. The dissolution rate is higher when fused silica is doped with germanium possibly due to the lower energy bonds of Ge-O and the presence of defects [13]. Germanium dioxide dissolution in HF may be described as,

$$
\mathrm{GeO}_{2}+6 \mathrm{HF} \rightarrow 2 \mathrm{H}_{3} \mathrm{O}^{+}+\mathrm{GeF}_{6}^{2-}
$$

The etching procedure applied to the optical fibers, consisted in dipping the tip of a graded index fiber in a solution of HF 48\% during 8 minutes. Due to the different etching rates of core and cladding, a concavity could be obtained in the fiber tip. Figure 1 and 2 show images, obtained by optical and electronic (SEM) microscopy, of the resulting cavities.

\subsection{Characterization of the FP cavities}

Fig. 1 shows the experimental setup used for characterization of the intrinsic FP cavities. The setup consisted in an optical broadband source with a central wavelength at $1550 \mathrm{~nm}$ and a bandwidth of $100 \mathrm{~nm}$. An optical circulator was used to redirect the reflected spectrum towards an optical spectrum analyzer (ADVANTEST Q8384) with a resolution of $10 \mathrm{pm}$ to observe the spectral response of the sensing head.In order to attain control on the cavity parameters, the progression of the etching process was monitored by recording periodically the cavity length and the external fiber diameter (estimated by optical microscopy). The results obtained can be seen in Fig. 2. As expected, the cavity length increases over the immersion time in HF, while the fiber diameter decreases. Although the HF reaction is much faster in the fiber core due to the presence of germanium, etching also takes place in the cladding region at a lower rate. For the graded index fiber with $62.5 \mu \mathrm{m}$ of core diameter, the rate of increase of the cavity length $(\Delta l / \Delta t)$ was 
$18.74 \pm 0.30 \mu \mathrm{m} / \mathrm{min}$, while the external fiber diameter was reduced with a rate $(\Delta d / \Delta t)$ of $-3.02 \pm 0.08 \mu \mathrm{m} / \mathrm{min}$. A finer adjustment of the cavity features can be attained by reducing the etching rates by simple dilution of the acid.

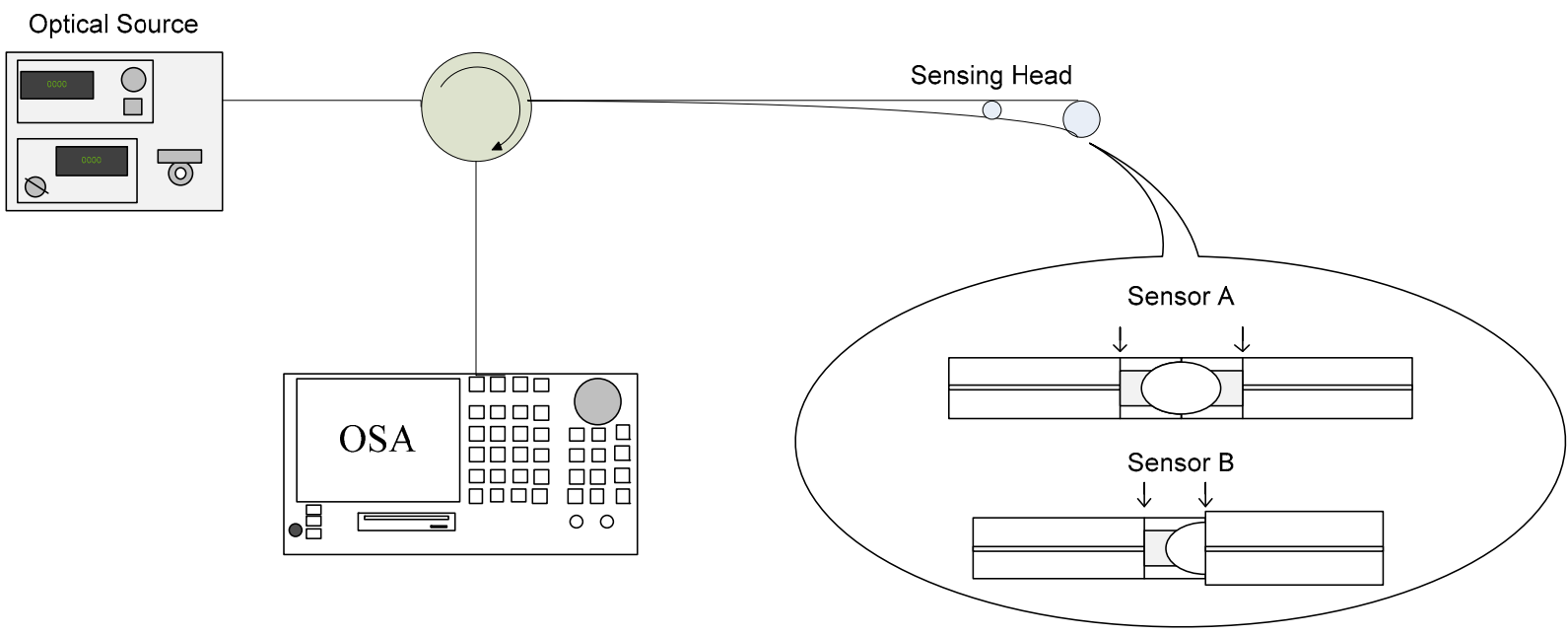

Fig. 1 - Experimental setup used for interrogation of the Fabry-Pérot cavity sensors.
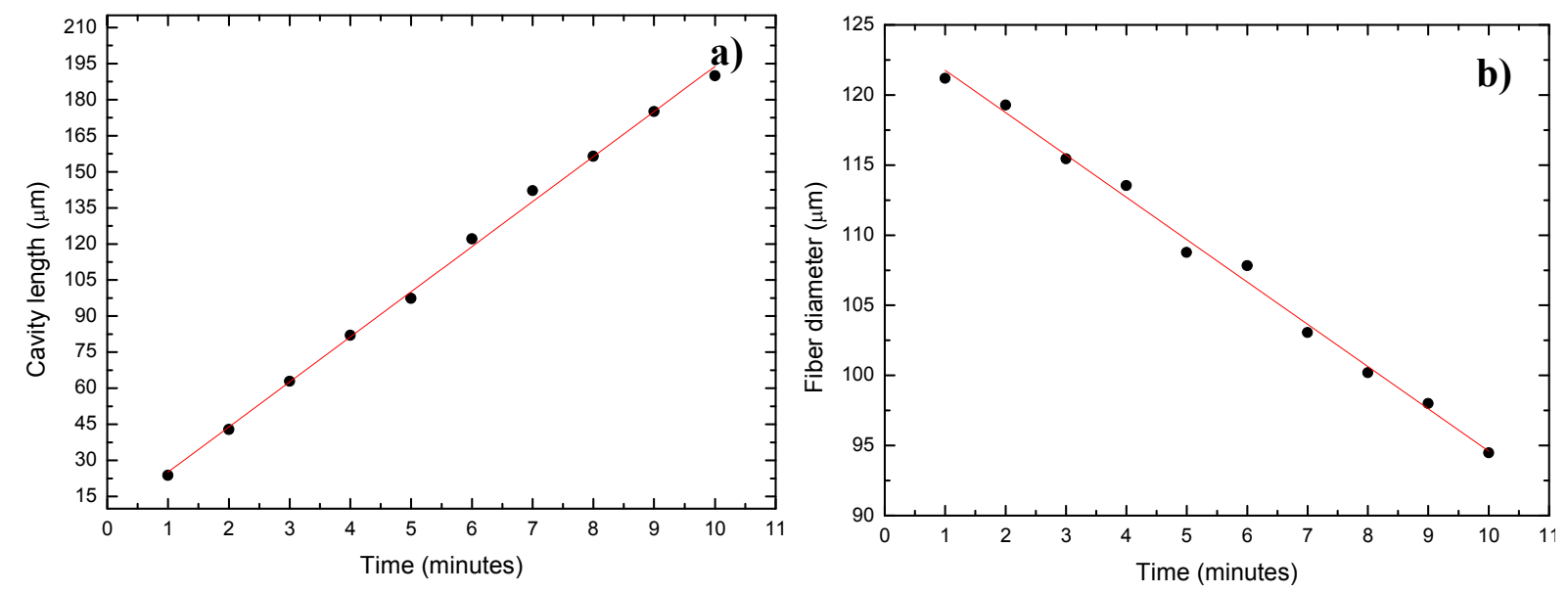

Fig. 2 - a) Evolution of cavity length with etching time; b) Evolution of the fiber diameter with etching time.

To create the intrinsic FP cavities, Fig. 3, a single mode fiber (SMF28) was spliced to a graded index fiber with $62.5 \mu \mathrm{m}$ of core diameter (GIF625) and then the GIF625 was cut the as near to the splice as possible, $\sim 150 \mu \mathrm{m}$ away from the splice (Fig. 3-1). The fiber was then chemically etched during 8 minutes creating a concavity, of approximately $100 \mu \mathrm{m}$, in the tip of the fiber (Fig.3-2). This process was repeated twice and then two identical concavities were spliced together creating sensor A (Fig.3-3). If a single concavity was spliced to a standard SMF28 fiber instead, sensor B was obtained (Fig.3-4). In Fig. 4 images obtained by optical microscopy of both sensors described above are shown. 
1)

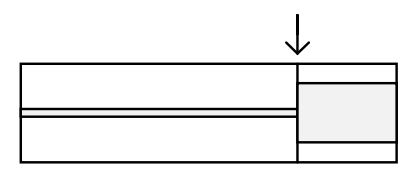

2)

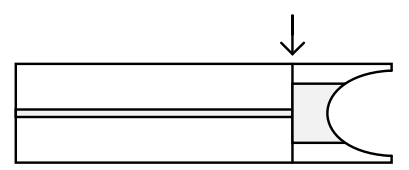

3)

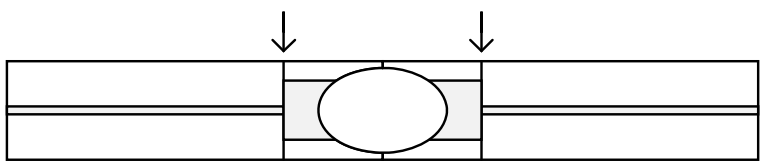

4)

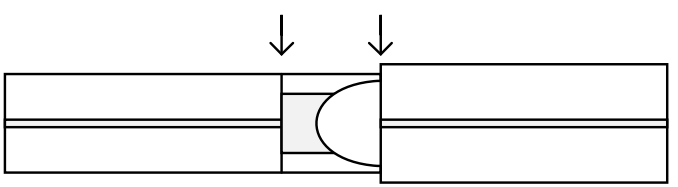

Fig. 3 - Schematic process of the sensors fabrication.
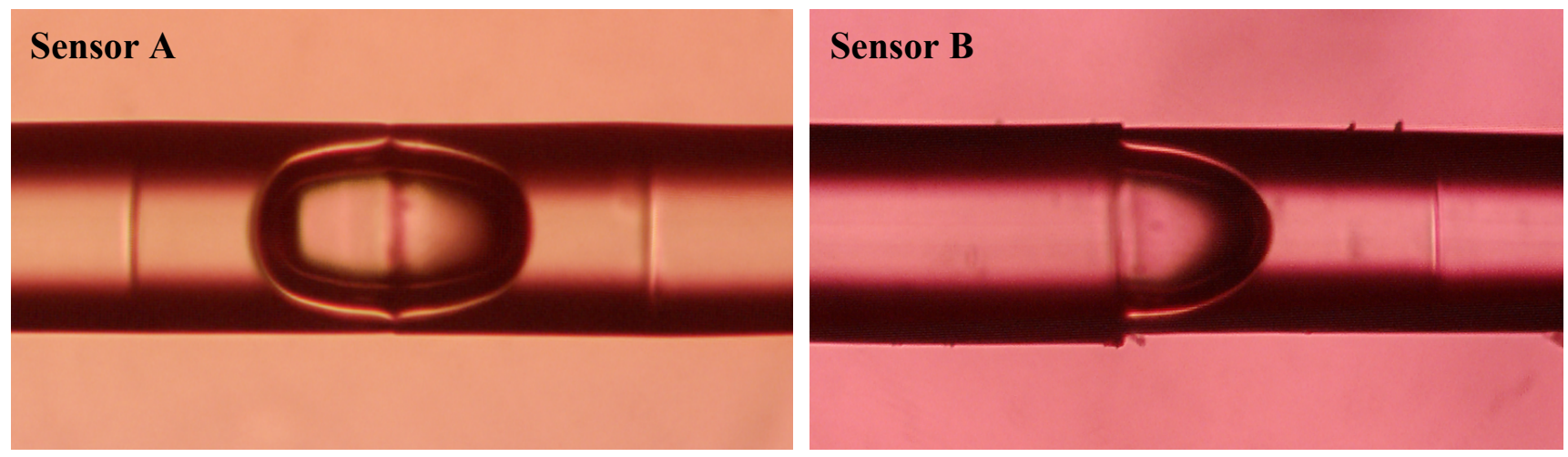

Fig. 4 - Optical microscopy images of sensor A (left) and sensor B (right).

Fig. 5 shows the spectra obtained in air for both cavities. A wavelength fringe period of approximately $8.75 \mathrm{~nm}$ can be observed for sensor B cavity while the fringe period for the sensor A cavity is $15 \mathrm{~nm}$. Because the FP spectral responses present a low finesse, its behavior can be approximated by a two wave interferometer. The differences in optical power are due to the higher loss in the longer cavity (sensor A). Sensor A displays fringes with a $6 \mathrm{~dB}$ contrast corresponding to a visibility of $75 \%$. On the other hand, in sensor B a fringe contrast of $2 \mathrm{~dB}$ can be measured that is equivalent to a visibility of $25 \%$. The visibility discrepancy is due to the differences in symmetry of the cavity interfaces in each configuration. 


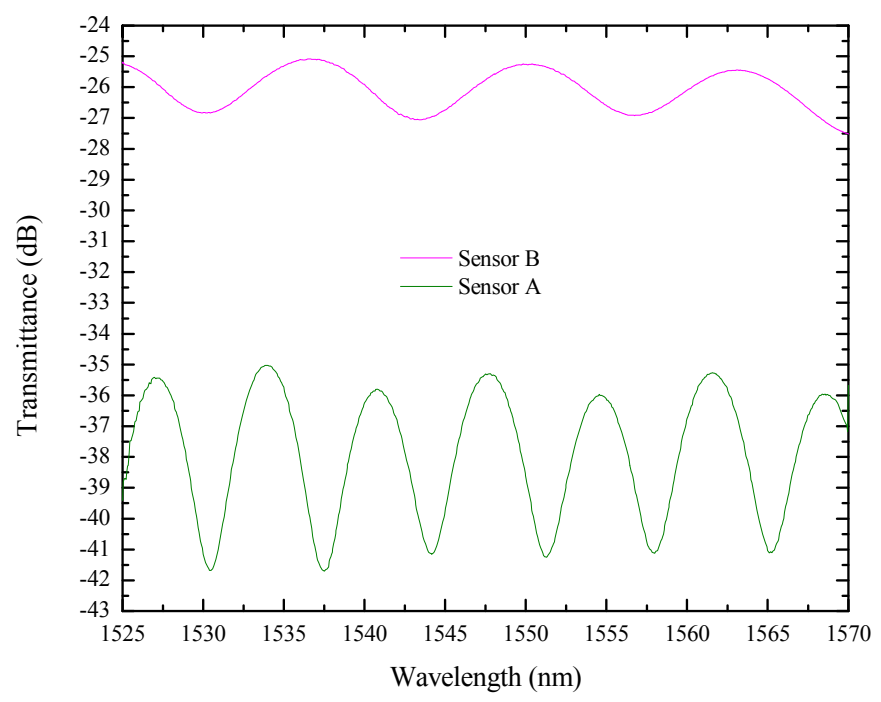

Fig. 5 - Output spectra of the cavities in air for sensor A and B.

\section{FP-CAVITY EXPERIMENTAL RESULTS}

\subsection{Response to Strain}

The spectral response of sensors A and B were monitored when the FP-Cavities were subjected to longitudinal strain. Fig. 6 shows the relationship between the wavelength response and the strain for both sensors. Sensor B showed a sensitivity of $3.14 \pm 0.05 \mathrm{pm} / \mu \varepsilon$ and sensor A a sensitivity of $1.85 \pm 0.07 \mathrm{pm} / \mu \varepsilon$. This disparity is expected due to the differences in the geometries created by chemical etching and splicing, i.e., the Young modulus is different for the sensor $\mathrm{A}$ and $\mathrm{B}$.

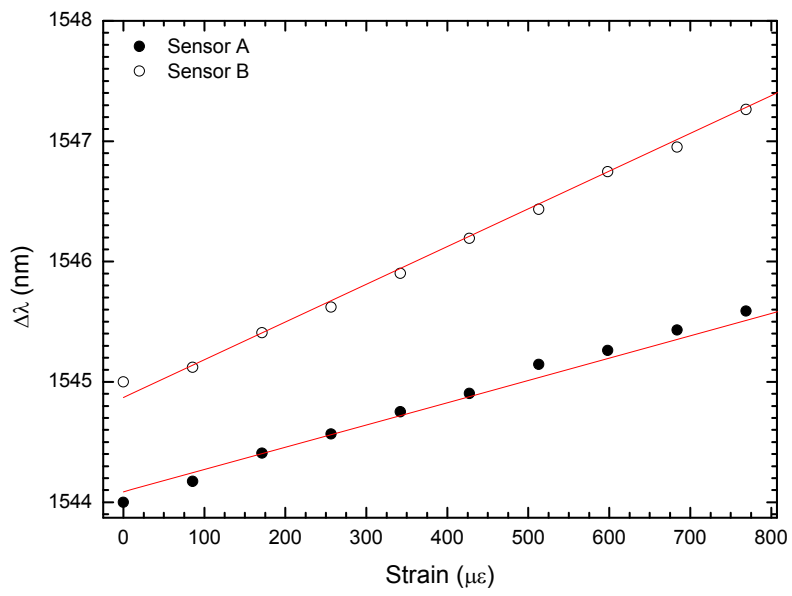


Fig. 6 - Strain response of the FP cavities of sensor A and B.

\subsection{Response to Temperature}

The intrinsic FP sensor response to high temperatures was characterized in a tubular oven. The characterization was realized between room temperature and $700{ }^{\circ} \mathrm{C}$ for the two sensors. Fig. 7 shows the wavelength shift of the spectral peaks in response to temperature. The sensors sensitivities are of $0.75 \pm 0.03 \mathrm{pm} /{ }^{\circ} \mathrm{C}$ and $0.98 \pm 0.04 \mathrm{pm} /{ }^{\circ} \mathrm{C}$ for sensors $\mathrm{A}$ and $\mathrm{B}$, respectively. The ratio between the two cavities length is 1.6 and the ratio between the two sensitivities is 1.3 . The difference is due to the symmetry and asymmetry of the sensor A and the sensor B, both in terms of their geometries and the types of fiber at each interface.

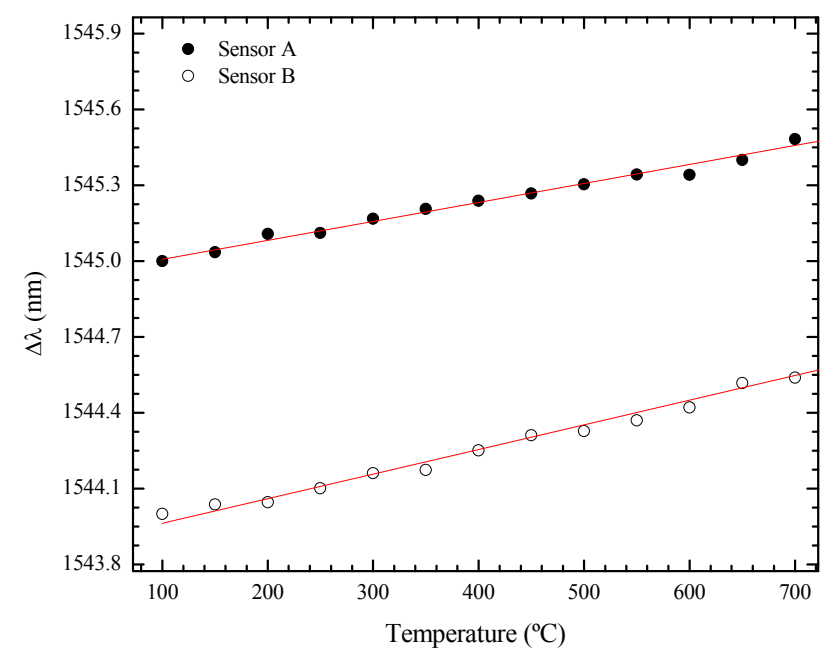

Fig. 7 - Temperature response of the FP cavities of sensor A and sensor B.

\section{CONCLUSIONS}

Two intrinsic FP optical fiber sensors were fabricated. The FP cavities were obtained by splicing a single mode fiber and a graded index fiber together, followed by a cut near to the splice, The short end of the fiber with graded index fiber was chemically etched with HF 48\% during 8 minutes and then the resulting cavity was A) spliced with an identical fiber and B) spliced to a single mode fiber. The application of chemical etching to a graded index fiber enables the fabrication of large cavity lengths with thin walls, when compared with single mode fiber, enabling higher sensitivity to longitudinal strain. Sensor A showed to be less sensitive to strain and temperature then Sensor B. Sensor A showed a sensitivity to temperature of $0.98 \pm 0.04 \mathrm{pm} /{ }^{\circ} \mathrm{C}$ and sensor $\mathrm{B}$ a sensitivity of $0.75 \pm 0.03 \mathrm{pm} /{ }^{\circ} \mathrm{C}$. As for longitudinal strain the sensitivities for sensor A and B were, respectively, of $1.85 \pm 0.07 \mathrm{pm} / \mu \varepsilon$ and $3.14 \pm 0.05 \mathrm{pm} / \mu \varepsilon$. Both structures are therefore suitable for the implementation of strain FP sensors for high temperature applications.

\section{REFERENCES}

[1] Rao, Y.-J., Recent progress in fiber optic extrinsic Fabry-Pérot interferometric sensors Optical Fiber Technology, 2006. 12: p. 227-237. 
[2] V. R. Machavaram, R.A.B., G.F. Fernando, Fabrication of intrinsic fiber Fabry-Pérot sensors in silica fibers using hydrofluoric acid etching. Sensors and Actuators A, 2007. 138: p. 248-260.

[3] Hae Young Choi, K.S.P., Seong Jun Park, Un-Chul Paek, Byeong Ha Lee, Eun Seo Choi, Miniature fiberoptic high temperature sensor based on a hybrid structured Fabry-Pérot interferometer. Optics Letters, 2008. 33(21): p. 2455-2457.

[4] Lee, C.E., Interferometric optical fiber sensors using internal mirrors. Electron. Lett., 1988. 24(4): p. 193194.

[5] Wei-Chung Du, X.-M.T., Hwa-Yaw Tam, Fiber Bragg Grating Cavity Sensor for Simultaneous Measurement of Strain and Temperature. IEEE Photonics Technology Letters, 1999. 11(1): p. 105-107.

[6] Brophy T. J., B.L.C., Shankar P. M., In-line singlemode fiber interferometer via concatenated biconical tapers. Electron. Lett., 1993. 29(14): p. 1276-1277.

[7] Susana F.O. Silva, O.F., Paulo Caldas, José L. Santos, F.M. Araújo, Luís A. Ferreira, Optical fibre refractometer based on a Fabry-Pérot interferometer. Optical Engineering, 2008. 47(5): p. 1-5.

[8] Z. L. Ran, Y.J.R., H. Y. Deng, X. Liao, Miniature in-line photonic crysral fiber etalon fabricated by $157 \mathrm{~nm}$ laser micromachinig. Optics Letters, 2007. 32(21): p. 3071-3073.

[9] Wei Tao, Y.H., Yanjun Li, Hai-Lung Tsai, Xiao Hai, Temperature-insensitive miniaturized fiber in-line Fabry-Perot interferometer for highly sensitive refractive index measurement. Optics Express, 2008. 16(8): p. 5764-5769.

[10] Gong, Y.A., et al., Fiber-Optic Fabry-Perot Sensor Based on Periodic Focusing Effect of Graded-Index Multimode Fibers. Ieee Photonics Technology Letters, 2010. 22(23): p. 1708-1710.

[11] Tafulo, P.A.R., et al., Intrinsic Fabry-Pérot cavity sensor based on etched multimode graded index fiber for strain and temperature measurement. IEEE Sensors Journal, 2011. Accepted for publication.

[12] Demagh, N.E., A. Guessoum, and H. Aissat, Chemical etching of concave cone fibre ends for core fibre alignment. Measurement Science \& Technology, 2006. 17(1): p. 119-122.

[13] Machavaram, V.R., R.A. Badcock, and G.F. Fernando, Fabrication of intrinsic fibre Fabry-Perot sensors in silica fibres using hydrofluoric acid etching. Sensors and Actuators a-Physical, 2007. 138(1): p. 248-260.

[14] Zhu, Y.Z. and A.B. Wang, Miniature fiber-optic pressure sensor. Ieee Photonics Technology Letters, 2005. 17(2): p. 447-449. 